\title{
Active mechanical properties of the smooth muscle of the urinary bladder
}

\author{
D. J. Griffiths* \\ R. van Mastrigt \\ W. A. van Duyl \\ B. L. R. A. Coolsaet
}

Departments of Biological \& Medical Physics and of Urology, Erasmus University, Rotterdam, The Netherlands

\begin{abstract}
Strips of pig bladder have been maximally stimulated in vitro at $37^{\circ} \mathrm{C}$ via electrodes placed in the muscle, in order, particularly, to measure the dependence of the resulting active force on the velocity of shortening and on length changes. The active isometric force and the passive viscoelastic force are approximately, but not precisely, additive. The active isometric force, like the steady (equilibrium) passive force, is a function of the extension of the strip above its rest length, which is increased after subjection to a high passive force. The steady passive force increases quasiexponentially with this extension, of which it is therefore a measure. The active isometric force $F_{i s o}$ increases approximately linearly with the extension until it approaches a maximum in the region where it and the steady passive force are comparable in size. The maximum is partly obscured by rest-length changes. The dependence of the active force $F$ on the speed of shortening of the strip has been measured in a new way, with a correction for passive viscoelastic effects. For a given strip the ratio $F / F_{i s o}$ is, approximately, a function of the contraction velocity only. The function is similar to that of the classical Hill equation but not identical, possibly for geometrical reasons. The results imply that a velocity parameter $v^{*}$. analogous to Hill's parameter $b$, is approximately constant for each strip, independent of changes of length and rest length.
\end{abstract}

Keywords_Force/length relationship, Force/velocity relationship, Plasticity, Smooth-muscle strips, Viscoelasticity

List of symbols

$a=$ force parameter in Hill equation, $\mathrm{N}$

$b=$ velocity parameter in Hill equation, $\mathrm{mm} / \mathrm{s}$

$F=$ active force during shortening, $\mathrm{N}$

$F_{i s 0}=$ active isometric force, $\mathrm{N}$

$F_{p s t}=$ steady (equilibrium) passive force, $\mathrm{N}$

$f_{1-4}()=$ functions of variables in parentheses

$l=$ length of muscle strip, $\mathrm{mm}$

$l_{0}=$ rest length of muscle strip, $\mathrm{mm}$

$v=$ speed of shortening, $\mathrm{mm} / \mathrm{s}$

$v_{\max }=$ physiological maximum speed of shortening, $\mathrm{mm} / \mathrm{s}$

$v^{*}=$ velocity parameter for muscle strip, $\mathrm{mm} / \mathrm{s}$

\section{Introduction}

DURING micturition the urinary bladder contracts from a volume of several hundred millilitres (in man) to essentially zero, actively expelling its contents. Correspondingly, the length of the muscle tissue changes greatly (CARPENTER, 1968). Therefore, in order to understand the course of micturition, it is necessary to know how the mechanical properties of the contracting muscle depend on changes in its

Received 23rd May 1978

"On leave from the Department of Physics, University of Exeter. Devon, England

$0140-0118 / 79 / 030281+10 \$ 01 \cdot 50 / 0$

(c) IFMBE : 1979 length. What is required is a series of simple approximations that can be used in a mathematical model of micturition (GRIFFITHS and Rollema, 1979).

Unstimulated, noncontracting bladder muscle behaves like a passive viscoelastic substance. It also exhibits plasticity: its rest length is increased after loading (CoOLSAET et al., 1976). We shall refer to forces exerted by unstimulated muscle as 'passive'.

When the muscle is stimulated to contract, an extra active force is developed, which presumably depends on the length of the muscle and on the speed of shortening as in other smooth and striated muscles (Gordon and Siegman, 1971). The active force decreases with increasing speed of shortening. The active force at constant length (zero speed of shortening) is called the isometric force. In order to make reliable measurements of the velocity dependence of the active force, both the passive viscoelastic force, which also is velocity dependent, and possible changes in the rest length must be taken into account.

In the experiments reported here the following have been investigated: the dependence of the isometric active force on changes in the length and rest length of the muscle; the relation between the isometric and passive forces; the form of the relation between the active force and the speed of shortening, 
and its dependence on changes of the length and rest length of the muscle. For the majority of the experiments strips of pig bladder were used. A few observations were made on human bladder. The strips were stimulated electrically by electrodes inserted in the muscle.

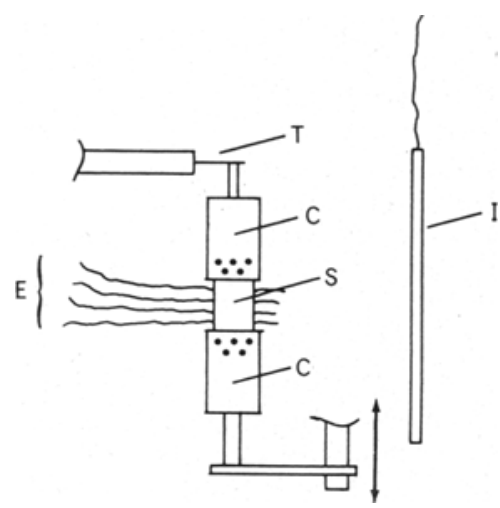

Fig. 1 Bladder strip (S), stimulating electrodes (E) and clamps $(C) . T=$ force transducer, $I=$ indifferent electrode. The strip is about $9 \mathrm{~mm}$ wide

\section{Material and apparatus}

Bladders of freshly killed pigs, male and female, were obtained from the local slaughterhouse. They were brought back to the laboratory at temperatures from $0^{\circ} \mathrm{C}$ to $37^{\circ} \mathrm{C}$ and were cut and mounted in the apparatus within 40 to $90 \mathrm{~min}$ of the death of the pig. Qualitatively similar results were obtained from all these strips, although those brought back in ice developed high active forces more reliably.
Each strip was about $15 \mathrm{~mm}$ long by $9 \mathrm{~mm}$ broad and was cut from the posterior wall, running longitudinally. The strip was fixed vertically between two toothed clamps (see CoOLsaEt et al., 1975) under very little tension $(\simeq 0.01 \mathrm{~N})$, so that about $8 \mathrm{~mm}$ of free length remained between the clamps. Thus the rest length of the portion of strip under investigation was initially about $8 \mathrm{~mm}$. This represented about $1 / 20$ of the total circumference of the bladder.

Electrodes made of silver wire, diameter $0.1 \mathrm{~mm}$, insulated except within the muscle, were threaded through the strip using a hypodermic needle as an inserter. They were approximately equally spaced and usually four in number, as shown in Fig. 1. The strip was then submerged in modified Krebs solution at $37^{\circ} \mathrm{C}$, oxygenated by bubbling with a $95 \% \quad \mathrm{O}_{2} / 5 \% \quad \mathrm{CO}_{2}$ gas mixture. During most experiments the bath was slowly perfused with fresh Krebs solution.

The two clamps formed part of a pneumatic straining and force-measuring apparatus that has already been described (CoOlsAET et al., 1976). The upper clamp was fixed to the cantilever of a force transducer, so that the force in the strip could be recorded on a chart recorder. The system was calibrated, and its linearity checked, with weights. The compliance of the measuring system was dominated by that of the force transducer, which was $0.1 \mathrm{~mm} / \mathrm{N}$. The lower clamp was attached to a flexible steel wire that ran over pulleys to the worm drive of an infusion pump. It was fixed also to a pneumatic piston, which kept the wire taut. Thus the lower clamp could be moved up and down and the strip shortened or lengthened at any one of a series of fixed speeds determined by the gearbox of

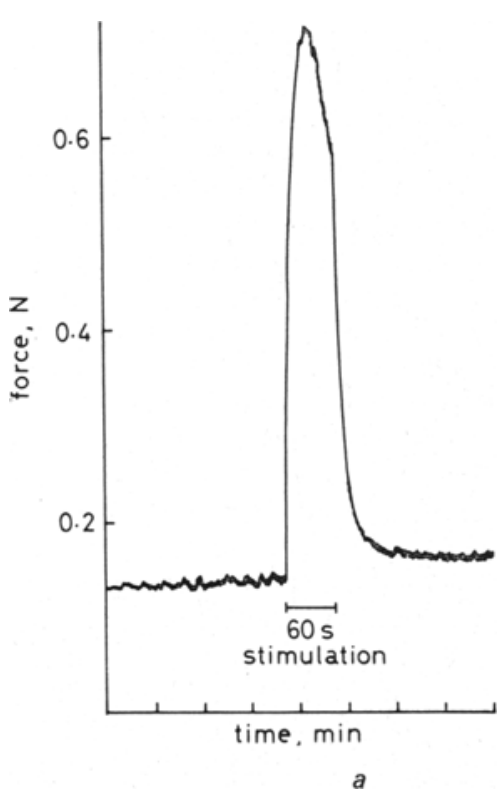

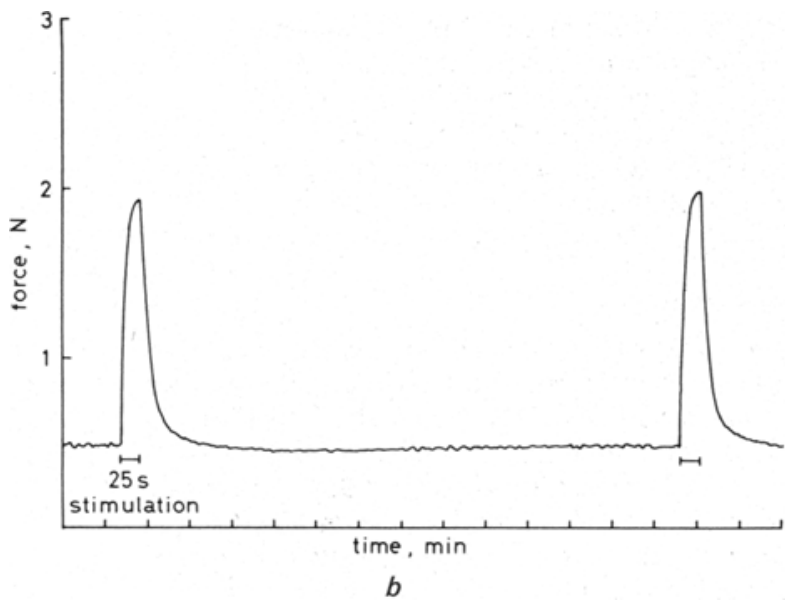

Fig. 2 Responses to stimulation by a train of electrical pulses ( $7 \mathrm{~ms}^{2}, 20 \mathrm{~s}^{-1}$ )

(a) Response to prolonged stimulation (60 s)

(b) Two successive responses to periods of stimulation just long enough (25s) for maximum (isometric) force to be registered 
the pump. Its position, and thus the length of the strip, was also recorded on the chart recorder.

For electrical stimulation of the strip, the stimulating electrodes were connected in parallel to three Grass S6 stimulators also in parallel. The 'earth' terminals of the stimulators were connected only to a large indifferent electrode, a strip of stainless steel, in the Krebs bath. The stimulators were synchronised and adjusted to give simultaneous pulses of the same duration and nominal voltage.

\section{Stimulation parameters}

The following results were established under isometric conditions:

(a) Stimulation by a train of electrical pulses gave a force that rose fairly rapidly to a inaximum and then decayed slowly (Fig. 2a). This maximum was taken as the isometric force.

(b) If stimulation was continued long after the maximum was!passed, the responses to succeeding periods of stimulation were reduced, apparently permanently. If, however stimulation ceased as soon as the maximum was reached (Fig. 2b), reasonably reproducible responses to similar successive periods of stimulation could be obtained, provided that a resting period of several minutes (usually $15 \mathrm{~min}$ ) was allowed between stimulations. The response varied slowly over a period of a few hours (Fig. 3), during which about 20 observations could be made.

(c) The active force was greater for pulses of negative than for those of positive polarity, with respect to the indifferent electrode. It was rather insensitive to the duration and frequency of the pulses, but was near maximal for a duration of $7 \mathrm{~ms}$ and a frequency of $20 \mathrm{~s}^{-1}$. These values were used in subsequent experiments.

(d) As the nominal voltage of the pulses was increased the response at first rose, but became

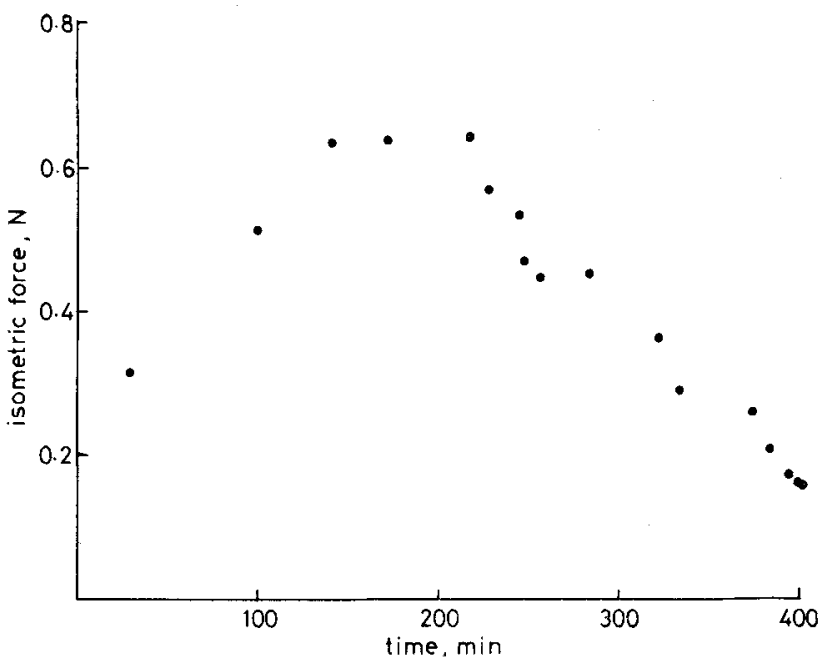

relatively constant above a certain voltage, whose value varied from strip to strip. This constant response was taken to be maximal. With four electrodes and three stimulators in parallel it appeared in experiments on several strips that a maximal response could always be expected if the nominal voltage was $50 \mathrm{~V}$. This value was used in subsequent experiments. If the nominal voltage was increased much above $50 \mathrm{~V}$ the strips were damaged, as evidenced by a rapid decrease in the response to successive stimulations. Since the stimulators were heavily loaded by the lowimpedance electrodes, the true voltage was only about $8 \mathrm{~V}$, corresponding to a stimulating current of about $250 \mathrm{~mA}$.

(e) Spontaneous rhythmic contractions (period $\simeq$ 30s) were superimposed on the 'passive' force exerted without stimulation. Their peak-peak amplitude never exceeded $10 \%$ of the active isometric force. They did not interfere with the measurements. Thus, under the conditions described, it was possible to make reasonably reproducible measurements of an active force that was maximal; i.e. was developed by contraction of the whole strip, or all of it that could be electrically stimulated.

\section{Measurements of active isometric force and passive} force

\subsection{Outline}

The passive viscoelastic force is velocity dependent, and, even at constant length, time dependent (CoolsaEt et al., 1975, 1976). Therefore, to obtain reproducible results it is simplest to make measurements at a given strip length when the passive force has become steady, after the time dependent part has decayed. The time dependent part is much smaller, and so decays to a negligible value more quickly, if the strip length is reduced rather than increased. 
Because the rest length of a strip is difficult to measure, different authors have defined and measured it in different ways (ANDERSON et al., 1968; Gordon and Siegman, 1971; CoOlsaEt et al.,
In some experiments two or more measurements of the isometric force were made at one length. Since the differences were small, in most experiments only one measurement was made at each length,

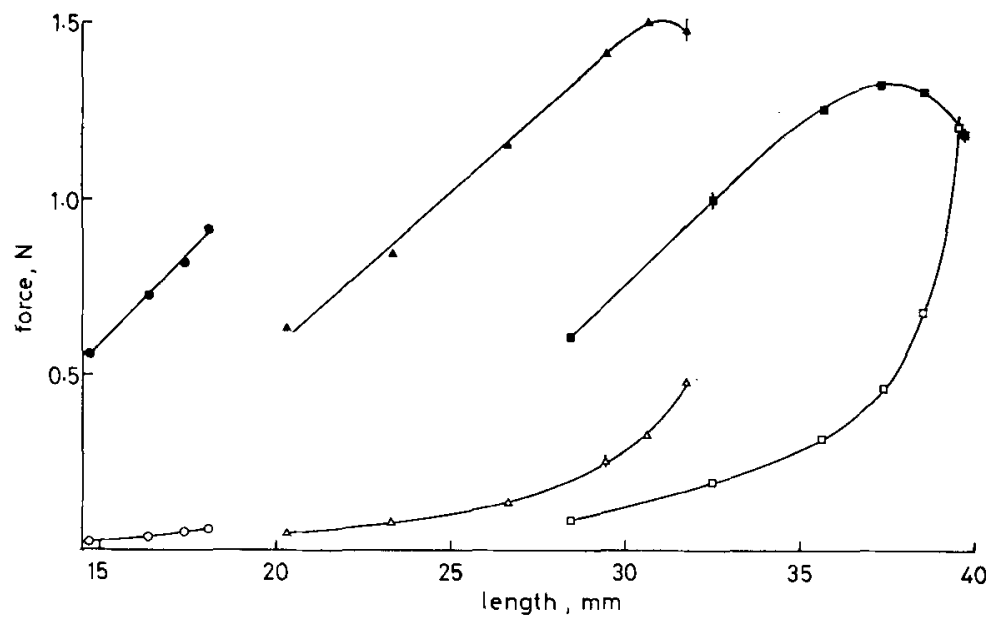

Fig. 4 Active isometric (black symbols) and steady passive (open symbols) forces as functions of length for one strip. Circles, triangles and squares $=$ respectively first, second and third series of measurements at decreasing lengths. Where a range is shown it indicates the difference between two successive measurements. Otherwise the difference was smaller than the size of the symbol. Curves linking the points are drawn purely to guide the eye

1976). However, changes in the rest length are easy to recognise. It tends to increase when the strip is stretched passively, especially if a high passive force is developed (CoolsaET et al., 1976; VAN MASTRIGT et al., 1978). If a series of measurements is made at increasing strip lengths, successively higher passive forces are generated every time the strip is lengthened and there is no means of controlling the increases in rest length. In a series of measurements at decreasing strip lengths, the passive forces are lower and tend to decrease, the rest length remains relatively constant and reproducible results are obtained.

Measurements were therefore made as follows. After mounting and immersion in the bath for about $\frac{1}{2} \mathrm{~h}$, the strip was extended at $0.6 \mathrm{~mm} / \mathrm{s}$ until a substantial passive force $(1-4 \mathrm{~N})$ developed, which was then allowed to decay viscoelastically. Measurements of the steady passive force and of the additional isometric tension developed during stimulation were made at a series of decreasing lengths. The strip was again extended, and further series of similar measurements made.

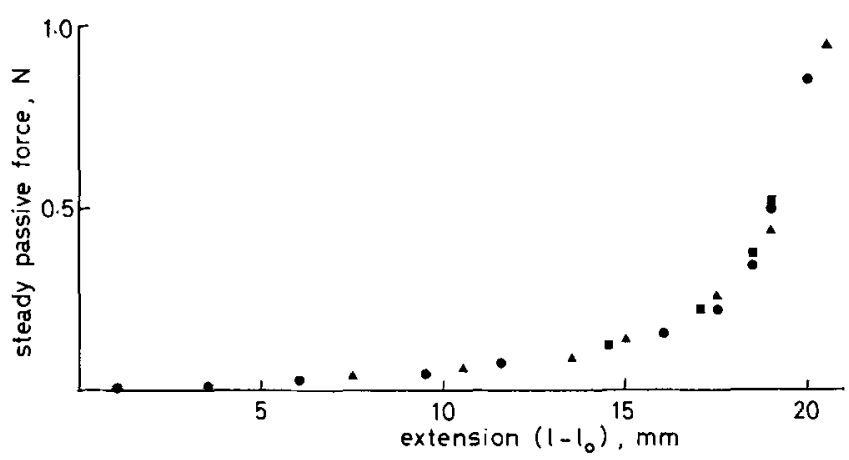

because of the limited number of measurements possible on one strip (see Section 3 part $b$ ).

\subsection{Results and discussion}

(a) Force/length measurements. Fig. 4 shows typical results from a strip from a pig bladder. Similar results were obtained from five other strips of pig bladder and from one human bladder strip.

The sets of points representing the active and passive forces are shifted further to the right in each series of measurements. This is a consequence of the increase in rest length, which is, for the last series, over $10 \mathrm{~mm}$. Since the initial rest length is only about $8 \mathrm{~mm}$ (see Section 2), the increase is very considerable.

The dependence of the steady passive force $F_{p s t}$ on the strip length in any one series, i.e. at constant rest length, is similar for each series, as shown in Fig. 5, where these passive force/length curves have been superimposed by shifting them along the length axis. Thus, as far as length dependence is concerned, and as a reasonable approximation, the 
steady passive force depends only on the extension $\left(l-l_{0}\right)$ relative to the rest length, and not on the rest length $l_{0}$ itself; i.e.

$$
F_{p s t} \simeq f_{1}\left(l-l_{0}\right)
$$

The function $f_{1}\left(l-l_{0}\right)$ has the expected quasiexponentially increasing form (ANDERSON et al., 1968; VAN MASTRIGT et al., 1978).

At constant rest length, the relation between the active isometric force $F_{i s o}$ and the strip length $l$ is quite different in form, see Fig. 4. It is nearly linear at small $l$, and possibly approaches a maximum at larger $l$, in the region where the passive force is high and comparable with the active force. This relation too is shifted along the length axis in different series of measurements, again suggesting a dependence on $\left(l-l_{0}\right)$. In addition, however, the overall magnitude of the active forces appears to decrease with increasing $l_{0}$. Therefore, provisionally

$$
F_{i s 0}=f_{2}\left\{\left(l-l_{0}\right), l_{0}\right\}
$$

(b) Relationship between active and passive forces. An important consequence of eqn. 1 is that the steady passive force, which is easy to determine, is a measure of the extension $\left(l-l_{0}\right)$, which is difficult to ascertain directly because of changes in $l_{0}$. To test eqn. $2 a$, then, the isometric force may be plotted against the steady passive force, as in Fig. 6. Each series of measurements (at a given $l_{0}$ ) yields one curve. The curves for the different series are qualitatively similar in shape, although quantitatively the isometric forces become a little smaller in each succeeding series, as one would expect from eqn. $2 a$ and Fig. 4. However, because of the time sequence of the measurements, these reductions in the isometric force could well be an artefact due to

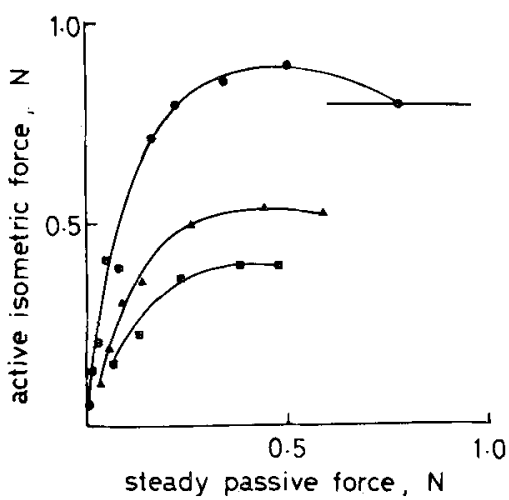

Fig. 6 Active isometric force as a function of steady passive force for one strip. Circles, triangles and squares = respectively first, second and third series of measurements at decreasing lengths. Range given for one point shows drift between two measurements due to rest-length change at high passive force. Curves are purely to guide the eye the time dependence of the active properties (Fig. 3), rather than a true dependence of the isometric force on the rest length.

In order to eliminate any such artefact, measurements were made on fresh strips as follows. The isometric force was measured at a given steady passive force. The strip was stretched to increase its rest length, shortened to regain approximately the same steady passive force, and the isometric force measured again. This was repeated several times. The result of such an experiment is shown in Fig. 7. Within experimental error the isometric forces are equal in five measurements at similar steady passive forces, although the strip length, and therefore the rest length, has increased by $16.5 \mathrm{~mm}$. Therefore, the apparent rest-length dependence of the active force in Fig. 6 is an artefact in this force range. Since in addition all the curves have a similar form

$$
F_{t s 0} \simeq f_{3}\left(F_{p s t}\right)
$$

independent of changes in the rest length, to a reasonable approximation. The form of this function is shown in Fig. 6.

Since $F_{p s t}$ is a measure of $\left(l-l_{0}\right)$ (eqn. 1$)$, eqn. $2 a$ can be rewritten as

$$
F_{i s 0} \simeq f_{2}\left(l-l_{0}\right) \text {. }
$$

i.e. to a first approximation the active isometric force, like the steady passive force, depends only on the extension $\left(l-l_{0}\right)$ and not directly on the rest length $l_{0}$ of the strip.

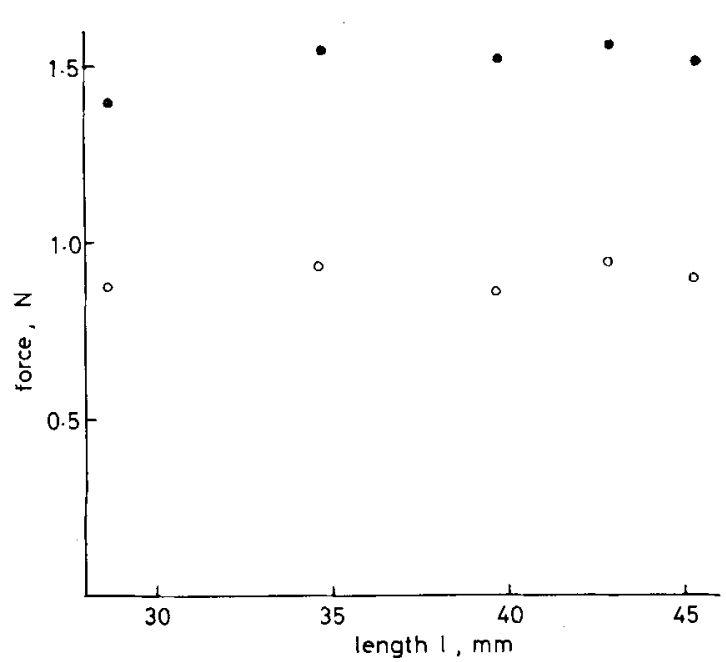

Fig. 7 Active isometric force (black circles) and steady passive force (open circles) as functions of length, for one strip. Passive force is approximately constant, but rest length has been increased between successive measurements, so that (total) length continually increases. Isometric force also remains approximately constant 
(c) Existence of a maximum in the isometric force/ length relationship. Previous authors, seeking a maximum in the active force/length relation for smooth muscle similar to that in striated muscle, have found that it occurs at high extensions where the passive and active forces are comparable (ANDERSON et al., 1968; Gordon and SIEGMAN, 1971), consistent with Figs. 4 and 6. However, it is difficult in this region to carry out measurements at constant rest length. If measurements are made at increasing strip lengths, any increase in the rest length as the presumed maximum is approached reduces the active force (see Fig. 4), and so can yield a spurious maximum. Indeed, the presumed maximum is difficult to reach at all by increasing the length of the strip, since increase of the rest length causes it continually to recede to greater lengths.

(d) Plasticity. Increases in rest length, as observed here and by VAN MASTRIGT (1977) appear to occur above a certain passive yield stress (VAN MASTRIGT et al., 19/8). They are not easily reversible. Occasionally what appeared to be a very slow, partial reversal was seen during these experiments, presumably either viscoelastic or metabolic in origin.

COOLSAET et al. (1976) have suggested that the rest length might decrease during active contraction. Neither the brief contractions produced by electrical stimulation, nor a sustained contraction produced by the addition of acetylcholine $(\simeq 1 \mu \mathrm{g} / \mathrm{ml})$ to the bath caused any significant change in rest length in these experiments.

(c) Additivity of passive and active forces. The isometric force and the steady passive force are additive by hypothesis, but the passive force is

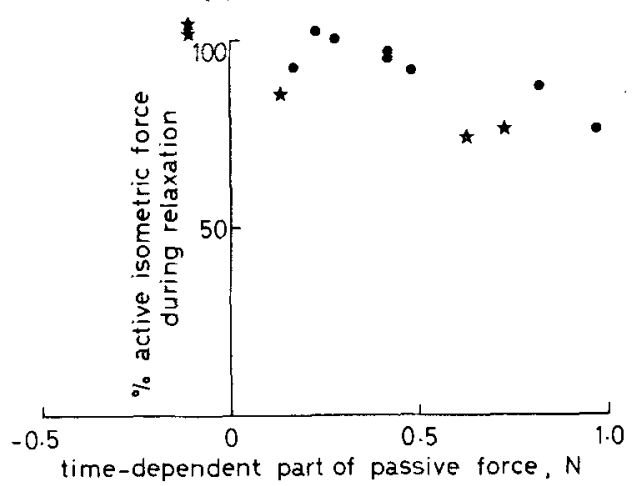

Fig. 8 The effect of passive viscoelastic relaxation on the active isometric force: results for two strips (circles and stars). The ordinate shows the isometric force during relaxation as a percentage of the isometric force after relaxation has ceased. The abscissa shows the difference between the passive force during relaxation and the steady passive force after relaxation: i.e. the time dependent part of the passive force. Positive values were obtained after extension, negative values after shortening. If the active and passive forces were exactly additive the ordinate would always be $100 \%$ viscoelastic in origin and in general varies in time. Experiments were done to see whether the isometric force was merely added to the time-dependent passive force, or whether it was altered when the passive force varied in time. These experiments were not conducted at large extensions, where even the 'steady' passive force changes with time because of the gradual increase of the rest length.

After a rapid increase in length, the isometric force was measured just after the increase, when the time dependent part was large and rapidly changing, and again considerably later when the time dependent part was negligible. The former measurement was smaller than the latter when the time-varying part of the passive force was large, see Fig. 8. Therefore the active and passive forces were not strictly additive, although the lack of additivity is not likely to be significant under most experimental circumstances.

After a rapid decrease in length the viscoelastic effects are much smaller and it was not possible to detect a significant alteration in the isometric force, as shown by the points at negative values of the abscissa in Fig. 8.

\section{Measurements of the active force developed during shortening of the strip}

\subsection{Outline}

The active force developed by a muscle depends not only on its length but also on its speed of shortening. In the past the speed of shortening was usually measured under a constant force. To reduce the length dependence and isolate the velocity dependence, the measurements were often made near the maximum of the isometric force/ length relation. With bladder muscle, difficulties arise:

(a) The maximum cannot easily be reached (Section 4.2)

(b) If one nevertheless works near the maximum, the passive force is large, velocity dependent and very sensitive to changes in length (see Fig. 5): thus, even if the total force on the muscle is constant, the passive part of it decreases rapidly as the muscle shortens and the active force correspondingly increases.

For these reasons the speed of shortening is not at all constant in such experiments, and it is difficult to obtain reliable results (GoRdon and SiEgMAN, 1971).

The velocity dependence has therefore been measured in a different way. The bladder strip was stimulated while it was shortening at a constant speed, determined by the Harvard pump (see Section 2). After the force had reached a maximum the shortening was stopped and the force rose further to its isometric value (see Fig. 9). The stimulation was then switched off so that the active force 
decayed, leaving the steady passive force. From these measurements one knows, for a given length and rest length: the steady passive force, the sum of the isometric force and the passive force, and the sum of the passive and active forces at a given speed of shortening. One could thus calculate the active and passive forces separately except for one difficulty: because of viscoelasticity, the passive force just after shortening is not equal to the steady value. However, after shortening the active and passive forces are essentially additive (Fig. 8). Therefore it is necessary, before or after these measurements, to shorten the strip in (ideally) a similar manner but without stimulation, and to record the subsequent viscoelastic relaxation. The steady passive force can then be corrected to yield its values just after shortening, so that the isometric force and the active force during shortening can be calculated, as shown in Fig. 9. Usually the viscoelastic correction is small compared to the active forces, so that high accuracy is not needed.

In some experiments the isometric force was measured again at the same length, after a few minutes, without shortening. Comparison of the two measurements confirmed that the method of correcting for the viscoelasticity was satisfactory.

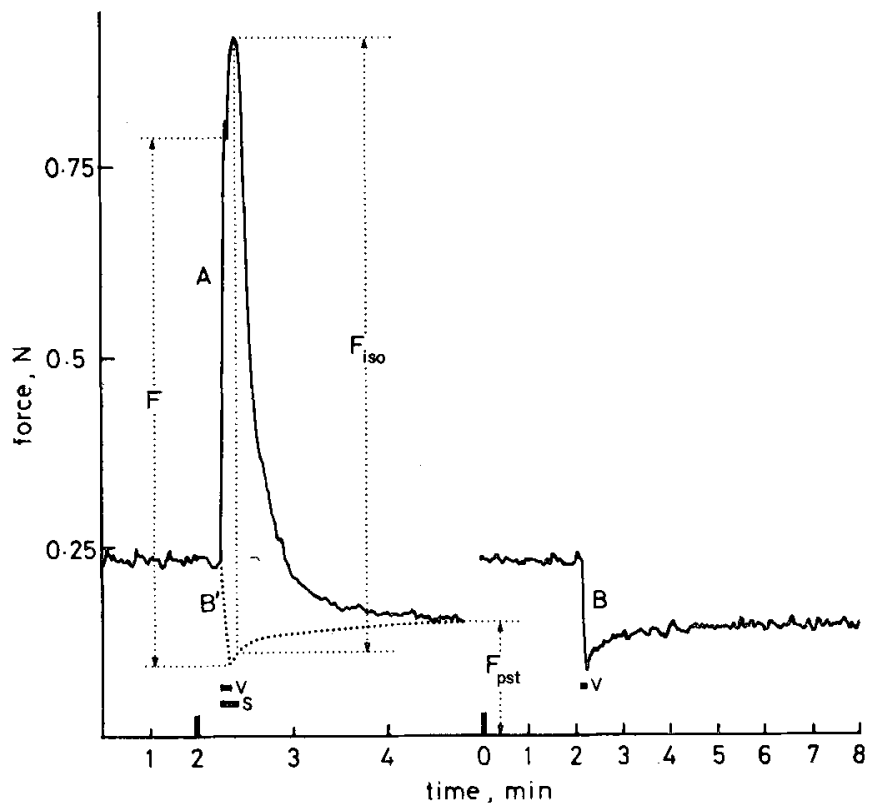

Fig. 9 Method of measuring of the velocity-dependence of the active force. A: The total force is measured during simultaneous stimulation and shortening at constant speed, at a certain length. Shortening is stopped and the isometric force is recorded at the same length. Stimulation is stopped and the steady passive force $F_{p s t}$ is measured at this length:

$B:$ Ideally, a precisely similar shortening without stimulation yields a passive viscoelastic relaxation curve, which enables correction of the passive force from its steady value to the values appropriate to the stimulation period. Thus the active force $F$ during shortening and the isometric active force $F_{i s}$ at the same length can be calculated and compared.

$V$ : periods of shortening. $S:$ period of stimulation. Dotted curve $B^{\prime}$ : passive relaxation curve $B$ displaced to coincide with stimulation measurement.

n.b. changes in time scale
Experiments of two principal types were carried out. In the first type measurements were made at a fixed speed of shortening, in order to determine the effects of changes of length and rest length on the relation between the active force and the velocity of shortening. This is of importance not only clinically, because the length of the bladder muscle one strip is made at various extensions and rest lengths. In the second type of experiment the actual form of the active force/velocity relationship was mermined, by measurements at different speeds of

\subsection{Measurements at a single speed of shortening}

The strip was extended at $0.6 \mathrm{~mm} / \mathrm{s}$ without Thulation. After the time dependent part of the with stimulation (in order to observe the active the way just described, Fig. 9) and without stimulation (in order to observe viscoelastic relaxation), until the active force became too small to be measured accurately. For such a series the rest length force during shortening and the isometric force in 
is essentially constant. The strip was then extended again and a second series of shortenings made, at a greater rest length, and so on. Since the viscoelastic relaxations and the active forces were observed at different lengths, the corrections for the passive viscoelasticity were estimated by interpolation, and the isometric force $F_{i s 0}$ and the active force $F$ during shortening calculated as shown in Fig. 9. The results for one strip are shown as functions of its length $l$ in Fig. 10.

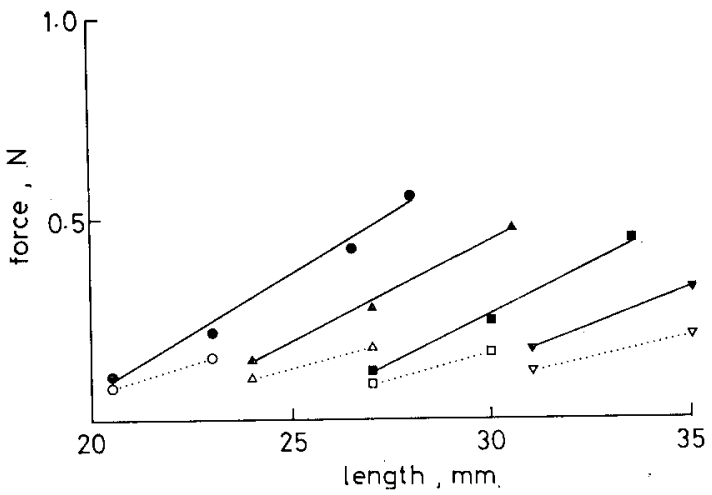

Fig. 10 /sometric force (b/ack symbo/s) and active force during shortening (open symbols) at a fixed speed $v=0.64 \mathrm{~mm} / \mathrm{s}$, as functions of length for one strip. Circles, triangles, squares, inverted triangles = respectively first, second, third and fourth series of measurements at decreasing lengths. Lines linking points are drawn purely to guide the eye

In order to make sense of these results, it is useful to express $F$ as a percentage of $F_{t s 0}$. Furthermore, since $F_{i s o}$ is a function not of $l$ but of the extension $\left(l-l_{0}\right)$ above the rest length, it is sensible

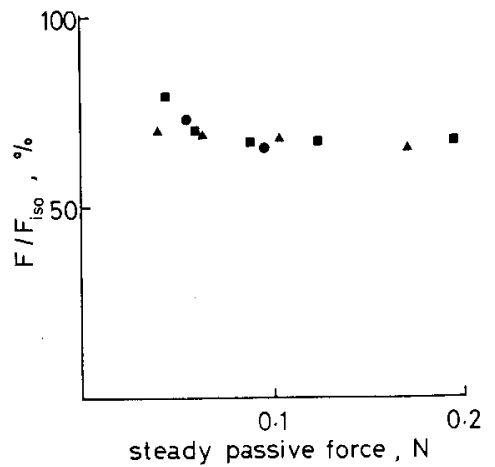

Fig. 11 Active force $F$ during shortening at a fixed speed $v=0.16 \mathrm{~mm} / \mathrm{s}$ expressed as a percentage of the corresponding isometric force $F_{\text {is } 0}$, plotted as a function of the steady passive force $F_{\text {pst }}$ in one strip. Circles, triangles, squares $=$ respectively first, second and third series of measurements at decreasing strip lengths to study the dependence of $F / F_{i s 0}$ on $\left(l-l_{0}\right)$, or more conveniently on $F_{p s t}$, the steady passive force, which is a measure of $\left(l-l_{0}\right)$, see eqn. 1 .

Results for one strip are shown in Fig. 11. Similar results have been obtained with four other strips from pig bladders and for one human bladder strip, at speeds of shortening so that, in different strips, $F / F_{i s 0}$ ranged from 40 to $80 \%$. As shown in Fig. 11, the values of $F / F_{i s 0}$ were very nearly the same in different series of shortenings, i.e. at different rest lengths. In most strips some dependence of $F / F_{l s 0}$ (expressed as a percentage) on $F_{p s t}$ was observed, ranging from about $+100 \% / \mathrm{N}$ to $-200 \% / \mathrm{N}$, with a mean of $-70 \% / \mathrm{N}$ for the pig-bladder strips (graphical estimates). Since the dependence was relatively slight, and varied in sign from strip to strip, it is reasonable to take $F / F_{i s o}$ as constant for a given $v$, independent of $F_{p s t}$ and of changes in the rest length.

Usually, after some hours of measurement, inconsistent results began to be obtained. These were ascribed to muscle damage and no further measurements were made.

Taken together, these results imply that, to a reasonable approximation

$$
F / F_{i s o} \simeq f_{4}(v) \quad . \quad . \quad . \quad . \quad . \quad . \quad . \quad .
$$

where the function $f_{4}(v)$ is, for a given strip, approximately independent of $l$ and $l_{0}$. Thus the velocity scaling of the force/velocity relation is unaffected by changes of length or rest length. In particular $v_{\text {max }}$, the physiological maximum speed of shortening, attained when $F / F_{t s o}=0$, should be approximately constant, independent of length changes, for a given strip. In fact, however, we shall see that $v_{\max }$

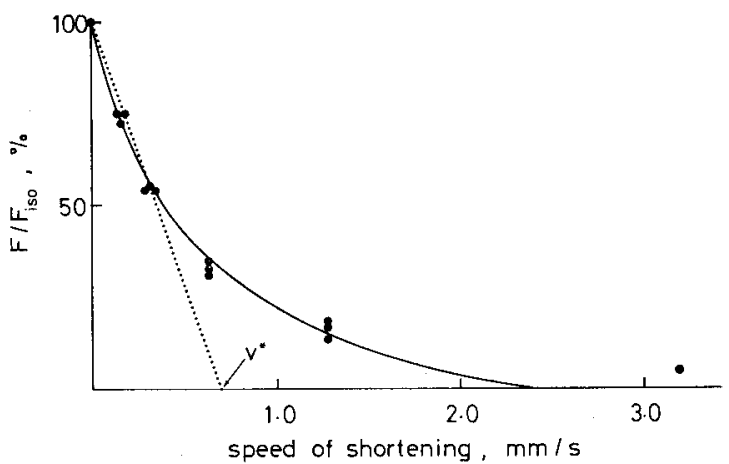

Fig. 12 Active force $F$ during shortening at speed $v$, expressed as a percentage of the corresponding isometric force $F_{\text {iso, }}$, plotted as a function of $v$. Points show experimental results for one strip. Curve shows classical Hill equation, eqn. 5, with $a / F_{i s 0}=0.25$ and $b=0.6 \mathrm{~mm} / \mathrm{s}$. Dotted line is a straight line fitted by eve to the experimental points for which $F / F_{i s 0}>$ $50 \%$. Its extrapolation to $F / F_{\text {iso }}=0$ yields the velocity parameter $v^{*}$, which here is equal to $0.7 \mathrm{~mm} / \mathrm{s}$ 
is not a good velocity parameter. A better parameter is proposed below: it too is approximately constant, independent of length changes.

\subsection{The form of the active force/velocity relationship}

Given eqn. 4, the form of the function $f_{4}(v)$ can be determined by measuring $F / F_{i s 0}$ at different $v$ on one strip. Such measurements have been made on six strips. The most reproducible results were obtained by use of the following method and by ensuring that the total number of stimulations of one strip did not exceed 12 .

The strip was extended at $0.6 \mathrm{~mm} / \mathrm{s}$ until the passive force was $1 \mathrm{~N}$. After the time dependent part had decayed, the strip was shortened at a given speed, with stimulation, as described in Section 5.1, to observe the force during shortening and the isometric force. The whole cycle of extension and shortening with stimulation was then repeated, with a different speed of shortening, and so on. Finally, a number of similar shortenings was made at the various speeds, but without stimulation, in order to correct for the viscoelastic effects (see Fig. 9). The values of $F / F_{i s o}$ obtained in this way for one strip are plotted against $v$ in Fig. 12. The reproducibility is satisfactory and the form of the function $f_{4}(v)$ (eqn. 4) can be seen.

The active force/velocity relationship for striated muscle is given by the well known HILL (1938) equation

$$
(F+a) v=\left(F_{i s 0}-F\right) b
$$

i.e.

$$
\left(F / F_{i s 0}+a / F_{i s 0}\right) v=\left(1-F / F_{i s 0}\right) b . . .
$$

where $a$ and $b$ are parameters characteristic of the muscle. $a / F_{\text {iso }}$ is typically 0.25 for striated muscle. The maximum speed of shortening $v_{\max }$ is attained when $F / F_{i s 0}=0$ and is given by $v_{\max }=F_{i s 0} b / a$ $(=4 b$, typically). Thus $b$ is a characteristic velocity parameter.

Eqn. 5, with $a / F_{0}=0 \cdot 25$, is also plotted in Fig. 12. The value of $b$ has been chosen so that the experimental points lie close to the curve in the region $F / F_{i s 0}>50 \%$. Although at first sight eqn. 5 is a reasonable fit to the points, one difference consistently found is that the experimental points lie on a curve with a long tail, extending to very high speeds of shortening at low nonzero values of $F / F_{t s 0}$. Therefore $v_{\max }$, the speed of shortening when $F / F_{i s 0}=0$, which has to be found by extrapolation, is very high, is not easy to determine accurately and is not representative of the behaviour of the muscle in the region $F / F_{i s 0}>50 \%$, which is clinically the most significant (GRIFFITHS, 1977). Furthermore, the high value of $v_{\max }$ may be due to a geometrical peculiarity of strips as opposed to complete bladders (see appendix). For all these reasons, $v_{\max }$ is not a useful velocity parameter for bladder strips. A better parameter $\left(v^{*}\right)$ is provided by the extrapolation to $F / F_{i s 0}=0$ of a straight line fitted to the experimental points in the region $F / F_{t s 0}>50 \%$, as shown in Fig. 12. $v^{*}$ is comparable, although not identical, with the parameter $b$ in the Hill eqn. 5. For five different strips the values of $v^{*}$ lay within $25 \%$ of $0.8 \mathrm{~mm} / \mathrm{s}$. For a complete pig bladder, where the speed of circumferential shortening is of importance, $v^{*}$ should be some $20 \times$ larger (see Section 2), i.e. $10-20 \mathrm{~mm} / \mathrm{s}$.

The principal conclusion of this section is that the $\%$-force/velocity relationship (eqn. 4) and the velocity parameter $v^{*}$ describing it are approximately constant for a given strip, independent of changes in the length and rest length.

\section{Conclusions}

The steady passive force exerted by a strip of bladder muscle is a useful measure of the extension $\left(l-l_{0}\right)$ above the rest length $l_{0}$. It is otherwise approximately independent of changes in the rest length.

The active isometric force $F_{i s 0}$ which is added to the steady passive force on maximal stimulation is also, to a first approximation, dependent only on $\left(l-l_{0}\right) . F_{i s o}$ rises approximately linearly from zero for small $\left(l-l_{0}\right)$, but approaches a maximum at large $\left(l-l_{0}\right)$, where it and the steady passive force are comparable in size.

The isometric active force and the steady passive force are by definition additive. If the passive force, which is viscoelastic, varies in time, small departures from additivity occur.

The increases in rest length that occur under high passive forces are not easily reversed. They make it difficult to observe the presumed maximum in the isometric force/length relationship.

The relationship between the active force $F$ and the speed of shortening $v$ is given by

$$
F / F_{i s o} \simeq f_{4}(v)
$$

where the function $f_{4}(v)$ is approximately unaffected by changes in $\left(l-l_{0}\right)$ and $l_{0}$. Although a decreasing function $v$, it differs in detail from that characteristic of striated muscle (the Hill equation), possibly for geometrical reasons. Nevertheless, a velocity parameter comparable to the Hill parameter $b$ can be defined, that is approximately independent of changes in the length and rest length of the strip.

Acknowledgments-D.J.G. is grateful to the Royal Society for the award of a European Fellowship and to Erasmus University Rotterdam for provision of facilities that enabled this work to be performed. 


\section{Appendix}

\section{Applicability of results to complete bladder}

In experiments on bladder strips the stress is uniaxial, unlike in a complete bladder, yet is sustained by muscle fibres running at many different angles to the axis. Among the consequences are the following:

(a) The passive and isometric force/length relations should be different in detail for strips (eqns. 1 and $2 a$ ) and for complete bladders. One would expect qualitative features, such as independence of rest-length changes, to be common to both.

(b) Plastic extension of the rest length may occur more readily in strips than in complete bladders, where certain shear-stress components are absent.

(c) The active force/velocity relationships should not be

\section{References}

Anderson, G. F., Pierce, J. M. and Blair, L. L. (1968) Tension changes in rabbit bladder muscle: effect of stretch. Investigative Urology, 6, 267-272.

Carpenter, F. G. (1968) Motor responses of bladder smooth muscle in relation to elasticity and fibre length. Investigative Urology, 6, 273-283.

Coolsaft, B. L. R. A., Van Duyl, W. A., Van Mastrigt, R. and Schouten, J. W. (1975) Viscoelastic properties of bladder wall strips. Investigative Urology, 12, 351-356.

Coolsaet, B. L. R. A., Van Mastrigt, R., Van Duyl, W. A. and Huygen, R. E. F. (1976) Viscoelastic properties of bladder wall strips at constant elongation. Investigative Urology, 13, 435-440.

Gordon, A. R. and Sregman, M J. (1971) Mechanical properties of smooth muscle, I-length-tension and force-velocity relations. Am. J. Physiol., 221, 12431249. identical for strips and for complete bladders Suppose, for example, that for individual muscle fibres, and thus for the complete bladder, no active force is developed above a shortening velocity $v_{\text {mux }}$ per unit length. When a bladder strip is shortened uniaxially at a speed greater than $v_{\max }$ per unit length, some oblique fibres are nevertheless shortening at speeds below $v_{\max }$ per unit length and thus exert an active force with an axial component. Therefore an active force is developed in the strip when the speed of shortening exceeds $v_{\max }$ per unit length: i.e. the apparent maximum speed of shortening is raised. Nevertheless qualitative features of the force/velocity relationship, such as independence of changes of length and rest length, should be common to strips and complete bladders.

GrIfFITHS, D. J. (1977) Urodynamic assessment of bladder function. Brit. J. Urology, 49, 29-36.

Griffiths, D. J. and Rollema, H. J. (1979) On the urine flow curves of healthy males: a mathematical model of bladder and urethral function during micturition. Med. \& Biol. Eng. \& Comput., 17, 291-300.

Hill, A. V. (1938) The heat of shortening and the dynamic constants of muscle. Proc. Royal Soc, B126, $136-195$.

Van Mastrigt, R. (1977) A systems approach to the passive properties of the urinary bladder in the collection phase. Thesis, Erasmus University Rotterdam.

Van Mastrigt, R., Coolsaet, B. L. R. A. and Van DUYL, W. A. (1978) Passive properties of the urinary bladder in the collection phase. Med. \& Biol. Eng., 16, 471-481. 